A. GUillaumin. - A propos D'Une tulipe monstrueuse.

les seuls qui semblent convenir au développement du Daphne Cneorum dans le Sud-Ouest de la France ${ }^{1}$.

\title{
A propos d'une Tulipe monstrueuse
}

PAR M. A. GUILLAUMIN.

Le genre Tulipa est décrit comme a uniflore, plus rarement bi ou triflore ; c'est qu'en effet certaines espèces ont normalement plusieurs fleurs comme le $T$. biflora, tandis que la majorité n'en ont normalement qu'une. Parmi ces dernières, la multiflorie n'est pas rare dans les espèces sauvages : $T$. saxatilis et surtout $T$. sylvestris, et on la connaît depuis plus de trois siècles dans les variétés horticoles issues du $T$. Gesneriana: il est fréquent, dit Krelage, dans sa variété Roi des Bleues ${ }^{2}$ et Bony en a même fixé une variété qu'il a appelée Monsieur S. Mottet $^{3}$.

La plante présentée aujourd'hui et qui a été remarquée par M. Gérôme, sous-directeur du Jardin d'Expériences du Muséum, dans une corbeille de cet établissement, appartient au $T$. suaveolens ou Tulipe Duc de Thol où cette monstruosité n'avait pas encore été signalée.

L'origine de la multiflorie est attribuée dans les ouvrages classiques de Tératologie à la fasciation seule : c'est évidemment le cas pour la plante présentée et pour la Tulipe Monsieur $S$. Mottet, mais une figure de Carrière ${ }^{4}$ montre qu'elle peut provenir de la ramification de la hampe; c'est également à la ramification que Labrie ${ }^{5}$ attribue les plusieurs fleurs observées par Verguin et lui chez le $T$. sylvestris.

Il y a donc lieu de distinguer les deux cas : fasciation et ramification.

1. Ce travail a été fait en collaboration avec M. J. Bouget, botaniste de l'Observatoire du Pic du Midi, qui m'a constamment aidé avec un dévouement auquel je mé plais à rendre hommage.

2. Revue horticole, 1882, p. $58, \mathrm{pl}$. col. et p. 550.

3. Ibid., 1909, p. 382-384, fig. 157.

4. Ibid., 1084, p. 57, fig. 14.

3. Actes de la Société linnéenne de Bordeaux, LXI, 1906, p. XLI-XLIII. 


\section{$2 \mathrm{BHL}$ Biodiversity Heritage Library}

Guillaumin, A . 1922. "A propos d'une Tulipe monstrueuse." Bulletin de la Société botanique de France 69, 213-213.

https://doi.org/10.1080/00378941.1922.10833425.

View This Item Online: https://www.biodiversitylibrary.org/item/93160

DOI: https://doi.org/10.1080/00378941.1922.10833425

Permalink: https://www.biodiversitylibrary.org/partpdf/161258

\section{Holding Institution}

Missouri Botanical Garden, Peter H. Raven Library

\section{Sponsored by}

Missouri Botanical Garden

\section{Copyright \& Reuse}

Copyright Status: Public domain. The BHL considers that this work is no longer under copyright protection.

This document was created from content at the Biodiversity Heritage Library, the world's largest open access digital library for biodiversity literature and archives. Visit BHL at https://www.biodiversitylibrary.org. 